\title{
An atypical acute exacerbation of COPD due to Aspergillus fumigatus
}

\author{
Cuneyt Tetikkurt ${ }^{1}$, Bahar Kubat ${ }^{1}$, Seza Tetikkurt ${ }^{2}$, Gulsum Karakas ${ }^{1}$ \\ ${ }^{1}$ Department of Pulmonary Medicine, Cerrahpasa Medical Faculty, Istanbul Cerrahpasa University, Istanbul; \\ ${ }^{2}$ Pathology Department, Florence Nightingale Hospital, Demiroglu Bilim University, Istanbul, Turkey
}

\begin{abstract}
A 64-year-old male with a history of stabile chronic obstructive pulmonary disease (COPD) presented with increasing dyspnea and sputum for the last two months. Complete blood count showed WBC 14x103/ml, Hgb: $14.2 \mathrm{~g} / \mathrm{dL}$ and eosinophilia. Blood biochemistry was normal. Chest $\mathrm{x}$-ray showed hyperlucency while thorax computed tomography (CT) revealed obstructive lung disease and bronchiectasis. Pulmonary function tests demonstrated severe obstructive lung disease and a negative bronchoreversibility with a moderately reduced diffusing capacity/alveolar volume (DLCO/VA). ABG gases revealed significant hypoxemia. Sputum culture was negative. Total IgE was $1140 \mathrm{ng} / \mathrm{ml}$. Aspergillus RAST, precipitins and aspergillus-
\end{abstract}

Correspondence: Prof. Cuneyt Tetikkurt, Department of Pulmonary Medicine, Cerrahpasa Medical Faculty, Istanbul Cerrahpasa University Tanzimat Sok. Serkan Apt. No: 8/16, 34728 Caddebostan, Istanbul, Turkey.

Tel. +90.216 .3601977 - Mobile: +90.532 .3810900$

Fax: +90.212 .5870217 .

E-mail: tetikkurt@gmail.com

Conflict of interest: All authors state explicitly that no potential conflicts exist.

Contributions: All the authors contributed equally. All the authors have read and approved the final version of the manuscript and agreed to be accountable for all aspects of the work.

Informed consent: Written informed consent was obtained from a legally authorized representative(s) for anonymized patient information to be published in this article.

Key words: ABPA; COPD; Aspergillus fumigatus; COPD acute exacerbation.

Received for publication: 17 August 2019.

Accepted for publication: 15 December 2019.

COpyright: the Author(s), 2020

Licensee PAGEPress, Italy

Monaldi Archives for Chest Disease 2020; 90:1155

doi: 10.4081/monaldi.2020.1155

This article is distributed under the terms of the Creative Commons Attribution Noncommercial License (by-nc 4.0) which permits any noncommercial use, distribution, and reproduction in any medium, provided the original author(s) and source are credited. galactomannan antigen were positive. CF genetic screening tests gave negative results. Allergic bronchopulmonary aspergillosis (ABPA) is a hypersensitivity reaction that occurs due to bronchial aspergillus colonization. It is most common in patients with asthma and cystic fibrosis. We present a COPD case with an acute exacerbation due to Aspergillus fumigatus that lead to an aberrant clinical profile unresponsive to conventional treatment. Clinicians should consider Aspergillus fumigatus as an etiologic agent in an atypical and severe COPD exacerbation.

\section{Introduction}

Allergic bronchopulmonary aspergillosis (ABPA) is a chronic pulmonary eosinophilic hypersensitivity reaction that leads to airflow obstruction and bronchiectasis. Disease results from an exaggerated allergic response to several fungal species, particularly Aspergillus fumigatus, which accounts for nearly $90 \%$ of the cases. The exact mechanism of ABPA is unknown. Several host factors and specific IgE-mediated type-I, IgG-mediated type-III and cell-mediated type-IV hypersensitivity reactions play a role in the pathogenesis of this disorder [1-6]. First described by Hinson et al., ABPA exclusively occurs in patients with underlying asthma $[7,8]$. While asthma is the most common and best understood contributing factor, ABPA may occur among those with cystic fibrosis and other underlying obstructive lung diseases. Although majority of patients with ABPA are associated with difficult asthma or cystic fibrosis, chronic obstructive pulmonary disease has been recognized recently as a risk factor for invasive aspergillosis. Airway colonization by Aspergillus species may occur as a common feature of chronic pulmonary diseases [9-12]. The lack of consensus regarding the diagnostic criteria may contribute to the difficulty for identifying this disorder and establishing its true prevalence among chronic obstructive pulmonary disease (COPD) patients. These underlying etiologies are crucial to identify since steroid treatment may lead to invasive fungal infection [13].

We present a case of severe exacerbation of a COPD patient in whom the etiologic agent for the acute exacerbation was identified as Aspergillus fumigatus. The case was a diagnostic dilemma as the symptoms of COPD and ABPA overlapped while the patient did not respond to conventional bronchodilator and antibiotic treatment in the long term with significant deterioration of his COPD profile. The only clue to the diagnosis was the appearance of recent blood eosinophilia followed by a high serum IgE level and positive immunologic markers thereafter. Clinicians should bear in mind that ABPA may be the etiologic factor in a patient with an aberrant acute COPD exacerbation that does not respond to conventional treatment. 


\section{Case Report}

A 64-year-old stabile COPD patient was admitted for progressive dyspnea, sputum, treatment failure and continuously worsening symptoms. The patient was an ex-smoker of 10 years with a 60 pack-year smoking history. Personal history consisted of COPD for six years without any other significant disease in the family history. On physical examination, there were rales in both lung bases. Blood pressure was $120 / 70 \mathrm{~mm} \mathrm{Hg}$ with normal heart sounds and a tachycardia of $114 /$ minute. Full blood count demonstrated a WBC: $11.4 \times 10^{3} / \mu 1$, eosinophilia $7.8 \times 10^{3} / \mu 1$, PLT $272 \times 10^{3} / \mu 1$ and Hgb $12.8 \mathrm{~g} / \mathrm{dL}$. The previous blood count three months before showed a normal eosinophil count $\left(3.6 \times 10^{3} / \mu 1\right)$. Serum biochemistry revealed CRP: $42.9 \mathrm{mg} / \mathrm{ml}$, creatinine: $0.38 \mathrm{mg} / \mathrm{ml}, \mathrm{BUN}: 14$ $\mathrm{mg} / \mathrm{dL}$, total protein: $5.2 \mathrm{gr} / \mathrm{dL}$, albumin: $3.2 \mathrm{gr} / \mathrm{dL}, \mathrm{AST}: 13 \mathrm{IU} / \mathrm{L}$, ALT: 16.7 IU/L, LDH: 204 IU/L, GGT: 24 IU/L and CK: 18 IU/L. ECG demonstrated a sinus tachycardia of $112 / \mathrm{min}$ and rare ventricular premature beats. Chest x-ray showed hyperlucency, fibrotic changes, bronchiectasis and minimal inflammatory infiltrations (Figure 1). Thorax CT revealed infiltrative lesions, fibrotic changes, proximal bronchiectasis, centrilobular emphysema and emphysematous bullae (Figures 1-3). Cystic fibrosis (CF) genetic screening was negative. Pulmonary function tests established severe obstructive lung disease revealing with a $\mathrm{FEV}_{1}: 1.83$ (45\%), a $\mathrm{FEV}_{1} / \mathrm{FVC}: 48 \%$ and $\mathrm{MEF}_{25-75}: 0.36$ (11\%). DLCO [Diffusing capacity (DLCO)] was $13.35 \mathrm{~mL} / \mathrm{mmHg} / \mathrm{min}(51 \%)$ and DLCO/VA [Diffusing capacity/alveolar volume (DLCO/VA)] was $15.28 \mathrm{~mL} / \mathrm{mmHg} / \mathrm{min} / \mathrm{L}$ (58\%). Bronchoreversibility was negative as $\mathrm{FEV}_{1}, \mathrm{FVC}$ and $\mathrm{MEF}_{25-75}$ revealed only $4 \%, 6 \%$ and $5 \%$ increase after $4 \times 100 \mu \mathrm{g}$ salbutamol inhalation. There was also no significant change of the lung function test values following one month of oral $32 \mathrm{mg}$ oral methylprednisolone treatment that justified the diagnosis of COPD. Arterial blood gases (ABG) exhibited a $\mathrm{pO}_{2}$ of $50 \mathrm{~mm} \mathrm{Hg}$, a $\mathrm{pCO}_{2}$ of $48 \mathrm{~mm} \mathrm{Hg}$ and a $\mathrm{pH}$ of 7.32 on room air. Sputum cytology and culture were negative. The patient had received brief courses of antibiotics and a long term combined inhaled steroid and long-acting beta agonist, ipratropium bromide and oral theophylline $600 \mathrm{mg} /$ day which lead to a brief response for his symptoms but the treatment was unsuccessful while the clinical manifestations continued to deteriorate. Complete blood count, quantitative immune globulin measurements and skin testing for delayed hypersensitivity were normal ruling out immunodeficiency. Serum total IgE was $1140 \mathrm{ng} / \mathrm{ml}$. This high above nor-

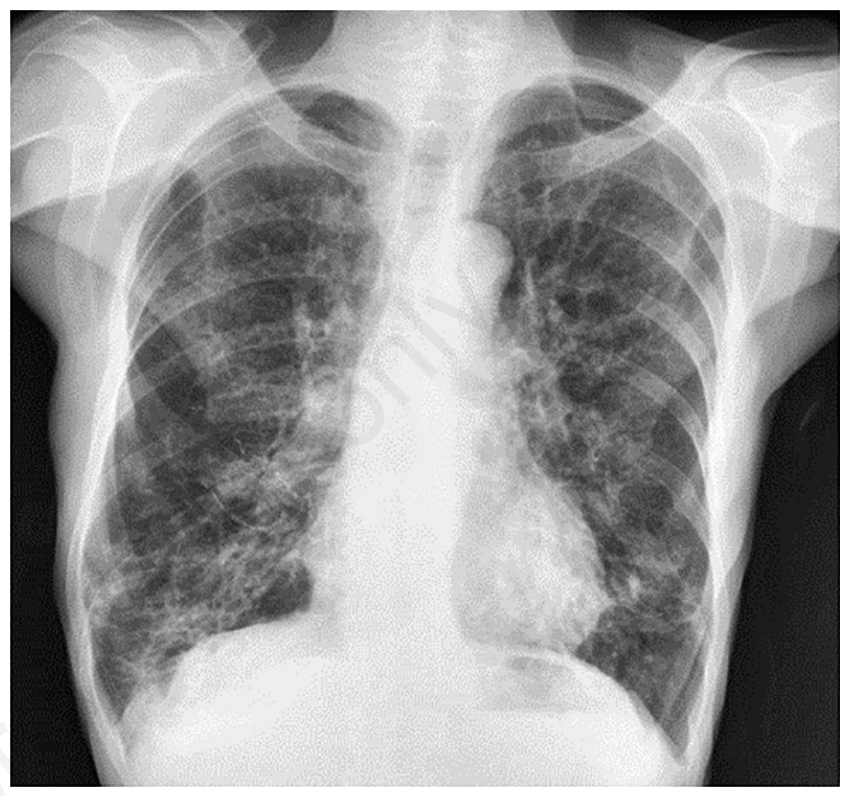

Figure 1. Chest $\mathrm{x}$-ray showing emphysema, bronchiectasis, fibrotic parenchymal changes and nodular opacities.
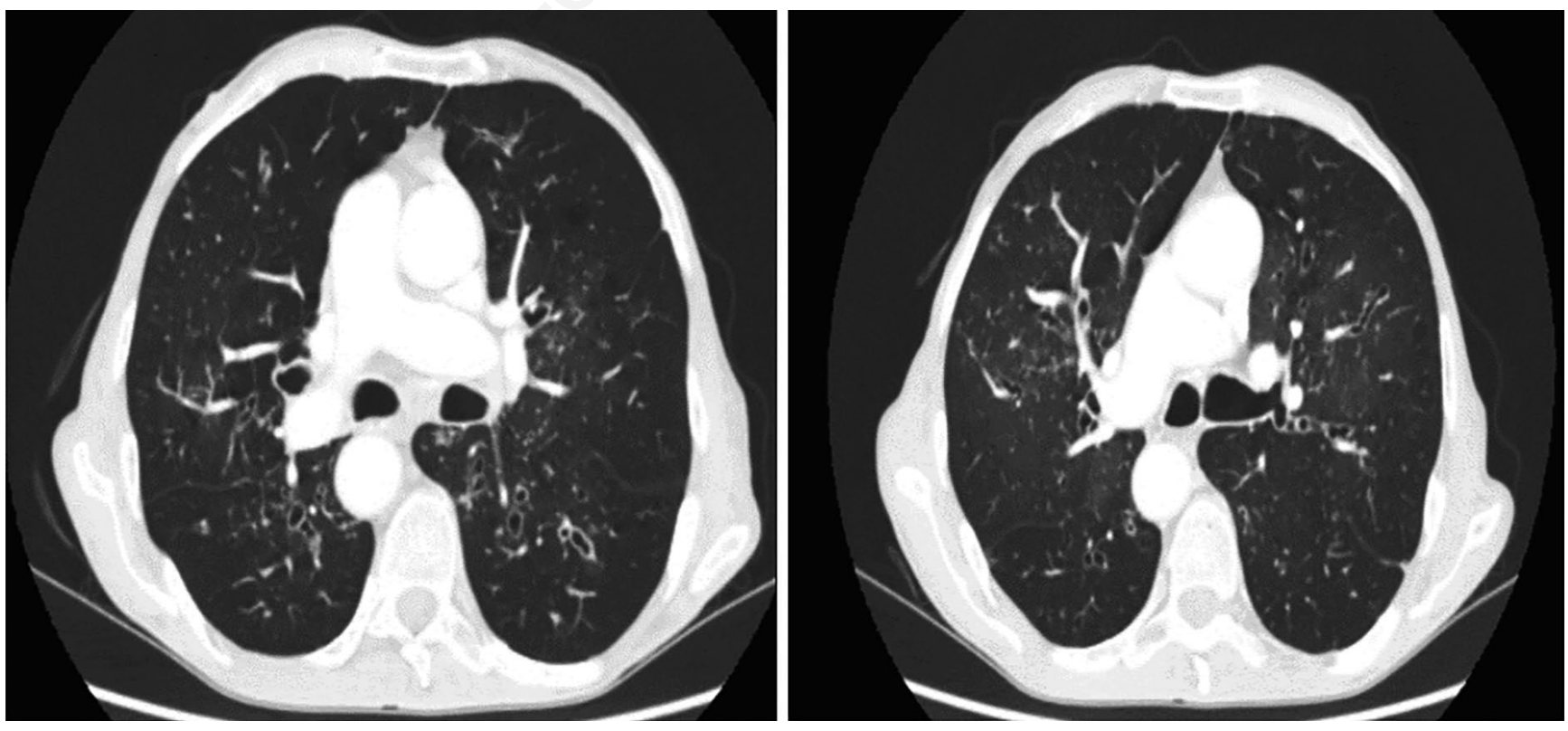

Figure 2. Thorax CT revealing diffuse bronchiectasis, centrilobular emphysema in the upper lobes and nodular infiltrations. 
mal value suggested that a hypersensitivity reaction was responsible for the deterioration of the patient's symptoms. Aspergillus radioallergosorbent test (RAST) and precipitins and aspergillusgalactomannan antigen were positive. The final diagnosis was an acute exacerbation of COPD due to Aspergillus fumigatus colonization. The patient was commenced on methylprednisolone 32 $\mathrm{mg}$ /day, a combined inhaled steroid and long-acting beta agonist, ipratropium bromide and oral theophylline $600 \mathrm{mg} /$ day with 4 $\mathrm{L} / \mathrm{min} \mathrm{O}_{2}$ nasal oxygen. Following treatment, the $\mathrm{pO}_{2}$ increased to $62 \mathrm{~mm} \mathrm{Hg}$ and the patient had complete resolution of symptoms. The only symptom was dyspnea during prolonged exertion. He is under follow-up with the same treatment protocol and the steroid dose was reduced to $24 \mathrm{mg}$ /day without any exacerbation of his symptoms for the last six months.

\section{Discussion}

ABPA is a complex hypersensitivity reaction that occurs in response to colonization of the airways with Aspergillus fumigatus. It occurs almost exclusively in patients with asthma or cystic fibrosis [1-4]. Repeated episodes of bronchial obstruction, inflammation, and mucoid impaction can lead to bronchiectasis, fibrosis, airflow obstruction and respiratory compromise. Proteolytic enzymes released by Aspergillus contribute to the development of bronchiectasis by damaging the walls of the airways $[5,6]$. The chronic pulmonary eosinophilic hypersensitivity reaction of ABPA leads to airflow obstruction and bronchiectasis in the long term. Disease results from an exaggerated allergic response to several fungal species, particularly the Aspergillus fumigatus, which accounts for approximately $90 \%$ of the cases. The exact pathologic mechanism for this complex hypersensitivity reaction is unknown. Specific IgE-mediated type-I, type-III and cell-mediated type-IV hypersensitivity reactions are thought to contribute to the pathogenesis of this disorder [1-4]. ABPA is a disease that exclusively emerges in those with underlying obstructive airways disorders such as asthma or cystic fibrosis. Novey has reported in a recent epidemiologic study that ABPA occurs between 0.25 and $11 \%$ in asthma while the incidence may be greater than $20 \%$ among poorly controlled asthma patients [14]. ABPA is a disease exclusively seen in those with underlying obstructive disorders while it is not a common pathogen in the COPD patients and its real incidence in this group is unknown $[11,12]$. We present this case as the presence of ABPA may cause an acute exacerbation of COPD that usually arises due to bacterial pathogens. The second crucial point is the overlap of clinical manifestations in both disorders with the same profile that may lead to a significant delay in diagnosis.

The difficulty in establishing the diagnosis of ABPA is likely multifactorial. Clinical presentations are inconsistent and are nonspecific. Symptoms may present in childhood while others may remain asymptomatic for decades with no real age distribution and disease may present more commonly in the fifth and sixth decades [6]. Most of the patients experience long-standing and often difficult asthma profile while others may experience mild symptoms or remain relatively asymptomatic [8]. The lack of consensus regarding the diagnostic criteria contributes to the difficulty in identifying ABPA. Coexistence of an obstructive disease further beclouds the diagnosis of ABPA, as it could not be clear whether the deterioration of clinical manifestations is caused by the underlying obstructive disorder or by the ABPA itself, which was the case in our patient. Another crucial point is that if two diseases coexist, they will further potentiate the deterioration of the clinical picture. The only clue to the diagnosis of ABPA was the recently emerging eosinophilia followed by the established presence positive RAST and galactomannan tests thereafter. Tong et al. has revealed that Aspergillus colonization in the lower respiratory tract of the COPD patients affected their clinical outcome and lead to clinical treatment dilemma. Bao has also referred to the fact that a reliable diagnosis was required for the diagnosis of Aspergillus in COPD patients to improve the prognosis $[15,16]$. Clinicians should be notified that Aspergillus fumigatus may be the etiologic agent for an aberrant exacerbation of a COPD patient. An unresponsive conventional treatment response for an acute exacerbation of COPD
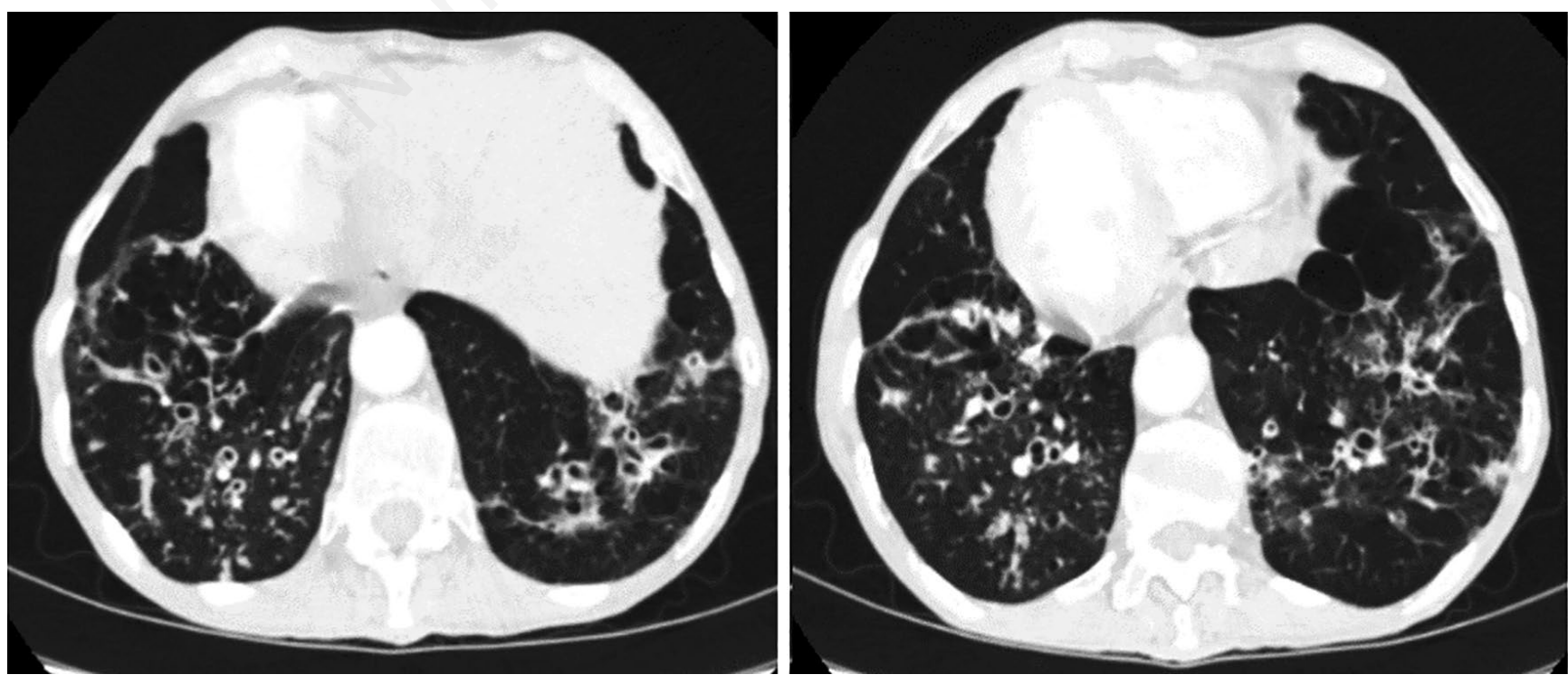

Figure 3. Thorax CT showing diffuse bronchiectasis, emphysematous bullae in the lower lobes and infiltrations. 
may also be the evidence for Aspergillus fumigatus as the pathologic agent in a chronic obstructive pulmonary disease patient. The recent development of eosinophilia in this COPD patient lead to ABPA suspicion while the subsequent RAST and galactomannan positivity confirmed the diagnosis of ABPA. The patient did not have asthma as verified by the lack of early or late bronchoreversibility and patient history revealed a smoking history with permanent dyspnea without acute dyspnea attacks dyspnea that may be relevant to asthma. A possible immunodeficiency status was ruled out by the presence of normal laboratory findings for the specific components of the immune system. Another crucial point relevant to this case was the absence of any significant radiologic infiltration implying infection despite the serious clinical manifestations and the critical COPD exacerbation. Absence of notable radiological findings in a patient presenting with such a severe and noteworthy clinical profile may point out to the presence of a probable Aspergillus infection.

\section{Conclusions}

ABPA is a chronic, progressive disorder affecting patients with airflow obstruction. Among patients with poorly controlled COPD, a diagnosis of ABPA should be considered. The prevalence of ABPA among COPD patients is unknown. Although the clinical manifestations are often nonspecific and usually overlap, several diagnostic criteria may be used to distinguish ABPA from obstructive diseases. The lack of definitive criteria for diagnosis and the overlap of the symptoms of the underlying primary disease with ABPA may lead to a significant delay in the detection of Aspergillus fumigatus as the responsible agent for the acute exacerbation of the primary obstructive disease. An aberrant acute exacerbation unresponsive to conventional treatment in a previously stabile COPD patient with a recently emerging eosinophilia should raise the likelihood Aspergillus as an etiologic agent. Absolutely disproportionate or absent infiltrative radiologic manifestations in a case presenting with such a severe clinical picture may also indicate an Aspergillus infection.

\section{References}

1. Greenberger PA. Allergic bronchopulmonary aspergillosis. J Allergy Clin Immunol 2002;110:685-92.

2. Zander DS. Allergic bronchopulmonary aspergillosis: an overview. Arch Pathol Lab Med 2005;129:924-8.

3. Tillie-Leblond I, Tonnel AB. Allergic bronchopulmonary aspergillosis. Allergy 2005;60:1004-13.

4. Agarwal R. Allergic bronchopulmonary aspergillosis. Chest 2009; 135:805-26.

5. Stevens DA, Moss RB, Kurup VP, et al. Allergic bronchopulmonary aspergillosis in cystic fibrosis--state of the art: Cystic Fibrosis Foundation Consensus Conference. Clin Infect Dis 2003;37:S225-64.

6. Greenberger PA, Patterson R. Allergic bronchopulmonary aspergillosis and the evaluation of the patient with asthma. J Allergy Clin Immunol 1988;81:646-50.

7. Hinson KF, Moon AJ, Plummer NS. Bronchopulmonary aspergillosis. Thorax 1952;7:317-33.

8. Glancy JJ, Elder JL, McAleer R. Allergic bronchopulmonary fungal disease without clinical asthma. Thorax 1981;36:345-9.

9. Bulpa P, Dive A, Sibille Y. Invasive pulmonary aspergillosis in patients with chronic obstructive pulmonary disease. Eur Respir J 2007;30:782-800.

10. Samarakoon P, Soubani A. Invasive pulmonary aspergillosis in patients with COPD: a report of five cases and systematic review of the literature. Chron Respir Dis 2008;5:19-27.

11. Guinea J, Torres-Narbona M, Gijón P, et al. Pulmonary aspergillosis in patients with chronic obstructive pulmonary disease: incidence, risk factors, and outcome. Clin Microbiol Infect 2010;16:870-7.

12. Ader F. Invasive pulmonary aspergillosis in patients with chronic obstructive pulmonary disease: an emerging fungal disease. Cur Infect Dis Rep 2010;12:409-16.

13. Eppinger TM, Greenberger PA, White DA, et al. Sensitization to Aspergillus species in the congenital neutrophil disorders chronic granulomatous disease and hyper-IgE syndrome. J Allergy Clin Immunol 1999;104:1265-72.

14. Novey JS. Epidemiology of allergic bronchopulmonary aspergillosis. Immunol Allergy Clin North Am 1998;18:641-53.

15. Bao Z, Chen H, Zhou M, et al. Invasive pulmonary aspergillosis in patients with chronic obstructive pulmonary disease: a case report and review of the literature. Oncotarget 2017;8:38069-74.

16. Tong X, Cheng A, Xu H, et al. Aspergillus fumigatus during COPD exacerbation: a pair-matched retrospective study. BMC Pulm Med 2018;18:55. 